\title{
Inferring efficient weights from pairwise comparison matrices.
}

\author{
R. Blanquero, E. Carrizosa and E. Conde \\ Facultad de Matemáticas, Universidad de Sevilla \\ Tarfia s/n, 41012 Seville, Spain \\ \{rblanquero, ecarrizosa, educon $\}$ @us. es
}

November 14, 2003

\begin{abstract}
Several Multi-Criteria-Decision-Making methodologies assume the existence of weights associated with the different criteria, reflecting their relative importance.

One of the most popular ways to infer such weights is the Analytic Hierarchy Process, which constructs first a matrix of pairwise comparisons, from which weights are derived following one out of many existing procedures, such as the eigenvector method or the least (logarithmic) squares. Since different procedures yield different results (weights) we pose the problem of describing the set of weights obtained by "sensible" methods: those which are efficient for the (vector-) optimization problem of simultaneous minimization of discrepancies.

A characterization of the set of efficient solutions is given, which enables us to assert that the least-logarithmic-squares solution is always efficient, whereas the (widely used) eigenvector solution is not, in some cases, efficient, thus its use in practice may be questionable.

Keywords: Analytic Hierarchy Process, Vector Optimization, Eigenvector method, Multiobjective Fractional Programming.
\end{abstract}

\section{Introduction}

Several strategies have been suggested in the literature to associate with a set $\mathcal{D}=\left\{d_{1}, \ldots, d_{N}\right\}$ of decisions weights $x_{1}, x_{2}, \ldots, x_{N}$ reflecting decision-maker's preferences. In the Analytic Hierarchy Process (AHP), [14. 15. 17. 18, an $N \times N$ matrix

\footnotetext{
${ }^{1}$ This research has been supported by the Spanish Science and Technology Ministry and FEDER Grants No. BFM2002-04525-C02-02 and BFM2002-11282-E.
} 
$A$,

$$
A=\left(\begin{array}{llll}
a_{11} & a_{12} & \cdots & a_{1 N} \\
a_{21} & a_{22} & \cdots & a_{2 N} \\
\vdots & \vdots & \ddots & \vdots \\
a_{N 1} & a_{N 2} & \cdots & a_{N N}
\end{array}\right)
$$

is obtained after asking the decision-maker (DM) to quantify the ratio of his/her preferences of one decision over another. In other words, for every pair of decisions $d_{i}, d_{j}$, the term $a_{i j}>0$ is requested satisfying

$$
a_{i j} \approx \frac{x_{i}}{x_{j}}
$$

The matrix $A$ so obtained must be a positive reciprocal matrix, i.e.,

$$
a_{j i}=\frac{1}{a_{i j}}>0 \quad \text { for all } i, j=1,2, \ldots, N .
$$

For a given positive reciprocal matrix $A$, different procedures can be followed in order to obtain weights $x_{1}, \ldots, x_{N}$ according to (1), see e.g. $\underline{11} \underline{\underline{2}} \underline{\underline{9}} \underline{\underline{13}}$ 16. In particular, Saaty proposes the so-called Eigenvector method (EM): $x$ is a column vector satisfying the equation

$$
A x=\lambda_{\max } x,
$$

where $\lambda_{\max }$ is the dominant eigenvalue of the positive reciprocal matrix $A$. See e.g. 15 for further details, and [5] for commercial software with it.

Many other choices have been proposed in the literature to derive $x$ according to (1), mostly given as optimal solutions of optimization problems such as

$$
\min _{x \in \mathbb{R}_{++}^{N}} \sum_{i, j=1}^{N}\left(\frac{x_{i}}{x_{j}}-a_{i j}\right)^{2},
$$

or

$$
\min _{x \in \mathbb{R}_{++}^{N}} \sum_{i, j=1}^{N}\left(\log \left(\frac{x_{i}}{x_{j}}\right)-\log \left(a_{i j}\right)\right)^{2},
$$

where $\mathbb{R}_{++}^{N}$ denotes the set of strictly positive vectors in $\mathbb{R}^{N}$.

It should become evident that different procedures, - (EM) or those derived from (2) or (3)-, although following (1), may yield different weights, and even different ranking of decisions may happen, as already shown e.g. in [16].

This naturally leads to the Nonconvex Vector-Optimization problem

$$
\min _{x \in \mathbb{R}_{++}^{N}}\left(\left|\frac{x_{i}}{x_{j}}-a_{i j}\right|\right)_{i \neq j}
$$

We recall the reader, e.g. $\underline{\underline{4}}$ 21, that, given an optimization problem $(P)$,

$$
\min _{x \in S}\left(f_{1}(x), \ldots, f_{k}(x)\right),
$$


$y \in S$ is said to dominate $x \in S$ if $f_{i}(y) \leq f_{i}(x)$ for all $i=1, \ldots, k$, with $f_{i}(y)<f_{i}(x)$ for some $i$. Moreover, $x \in S$ is said to be efficient for $(P)$ if no $y \in S$ dominates $x$, and $x$ is said to be locally efficient for $(P)$ if there exists a neighborhood $V$ of $x$ in $S$ such that no $y \in V$ dominates $x$.

Our aim is to find a full description of the set of locally efficient and efficient solutions for $(X)$, and to explore whether the usual weighting methodologies, are (or are not) efficient for $(X)$.

\section{A test for efficiency}

Problem $(X)$ is a multiple-objective nonlinear nonconvex problem whose feasible set is the strictly positive orthant $\mathbb{R}_{++}^{N}$, which is not closed. This makes at first glance $(X)$ very hard to solve. However, it is easy to construct an LP-based test of efficiency. Indeed, one has

Theorem 1 Let $x^{*} \in \mathbb{R}_{++}^{N}$. For each $k, l=1, \ldots, N$, define $\varepsilon_{k l}=\left|\frac{x_{k}^{*}}{x_{l}^{*}}-a_{k l}\right|$. Then $x^{*}$ is efficient for $(X)$ if and only if for each $k, l=1, \ldots, N, k \neq l, \varepsilon_{k l}$ is the optimal value of the Linear Problem

$$
\begin{array}{ll}
\min & t \\
\text { s.t. } & x_{i}-\left(\varepsilon_{i j}+a_{i j}\right) x_{j} \leq 0 \text { for all pairs }(i, j) \neq(k, l) \\
& x_{i}+\left(\varepsilon_{i j}-a_{i j}\right) x_{j} \geq 0 \text { for all pairs }(i, j) \neq(k, l) \\
& x_{k}-t \leq a_{k l} \\
& x_{k}+t \geq a_{k l} \\
& x_{l}=1 \\
& x_{1}, \ldots, x_{N} \geq 0 \\
& t: \text { unrestricted. }
\end{array}
$$

\section{PROOF}

It is a well known result of Vector Optimization, e.g. 4, that $x^{*}$ is efficient for $(X)$ if and only if for any pair of indices $k, l=1, \ldots, N, k \neq l, x^{*}$ is an optimal solution to the fractional optimization problem $\left(P_{k l}\right)$, [19]

$$
\begin{array}{ll}
\text { inf } & \left|\frac{x_{k}}{x_{l}}-a_{k l}\right| \\
\text { s.t. } & \left|\frac{x_{i}}{x_{j}}-a_{i j}\right| \leq \varepsilon_{i j} \text { for all pairs }(i, j) \neq(k, l) \\
& x_{1}, \ldots, x_{N}>0 .
\end{array}
$$

Let $k, l \in\{1, \ldots, N\}, k \neq l$ be given, and define the vector $y$ as $y=\frac{1}{x_{l}^{*}} x^{*}$.

Then, $x^{*}$ solves $\left(P_{k l}\right)$ if and only if $y$ does, what happens if and only if $y$ solves $\left(\hat{P}_{k l}\right)$,

$$
\begin{array}{ll}
\text { inf } & \left|x_{k}-a_{k l}\right| \\
\text { s.t. } & \left|\frac{x_{i}}{x_{j}}-a_{i j}\right| \leq \varepsilon_{i j} \text { for all pairs }(i, j) \neq(k, l) \\
& x_{l}=1 \\
& x_{1}, \ldots, x_{N}>0 .
\end{array}
$$


Problem $\left(\hat{P_{k l}}\right)$ is equivalent to the Linear Problem

$$
\begin{array}{ll}
\text { inf } & t \\
\text { s.t. } & x_{i}-\left(\varepsilon_{i j}+a_{i j}\right) x_{j} \leq 0 \text { for all pairs }(i, j) \neq(k, l) \\
& x_{i}+\left(\varepsilon_{i j}-a_{i j}\right) x_{j} \geq 0 \text { for all pairs }(i, j) \neq(k, l) \\
& x_{k}-t \leq a_{k l} \\
& x_{k}+t \geq a_{k l} \\
& x_{l}=1 \\
& x_{1}, \ldots, x_{N}>0 .
\end{array}
$$

This problem can also be written equivalently by replacing the strict inequalities $x_{j}>0$ by non-strict inequalities $x_{j} \geq 0$. In other words, we claim that $(5)$ is equivalent to (4). Indeed, any $x$ feasible for (5) is also feasible for (4). Conversely, for any $x$, feasible for (4), we have that

$$
\begin{array}{ll}
x_{l} & =1>0 \\
x_{l}-\left(\varepsilon_{l j}+a_{l j}\right) x_{j} & \leq 0 \text { for all } j,
\end{array}
$$

from which we deduce that $x_{j}>0$ for all $j$ : else, if $x_{j}=0$, (6) would yield $x_{l}=0$, which contradicts $x_{l}=1$. Hence, the result follows.

Although Theorem 1 enables us to check whether a given $x^{*} \in \mathbb{R}_{++}^{N}$ is efficient or not, it does not give insight in the structure of the efficient set. For this reason we devote the remaining of this section to provide alternative characterizations of efficiency for $(X)$.

Given a function $\pi: \mathbb{R}_{++} \longrightarrow \mathbb{R}$, consider the Vector-Optimization Problem $\left(X_{\pi}\right)$,

$$
\min _{x \in \mathbb{R}_{++}^{N}}\left(\left|\pi\left(\frac{x_{i}}{x_{j}}\right)-\pi\left(a_{i j}\right)\right|\right)_{i \neq j}
$$

Lemma 2 Let $\pi: \mathbb{R}_{++} \longrightarrow \mathbb{R}$ be strictly increasing. Then, $x^{*} \in \mathbb{R}_{++}^{N}$ is efficient for $\left(X_{\pi}\right)$ iff $x^{*}$ is locally efficient for $\left(X_{\pi}\right)$.

\section{PROOF}

For each $i, j=1, \ldots, N, i \neq j$, the function $x \in \mathbb{R}_{++}^{N} \longmapsto \frac{x_{i}}{x_{j}}$ is quasimonotonous and strictly quasimonotonous, $\underline{\underline{10}}$ i.e., both lower and upper level sets and strict lower and upper level sets are convex. Since $\pi$ is strictly increasing, the function $x \in \mathbb{R}_{++}^{N} \longmapsto \pi\left(\frac{x_{i}}{x_{j}}\right)$ is also quasimonotonous and strictly quasimonotonous. Hence, the function $x \in \mathbb{R}_{++}^{N} \longmapsto \max \left\{\pi\left(\frac{x_{i}}{x_{j}}\right)-\pi\left(a_{i j}\right),-\pi\left(\frac{x_{i}}{x_{j}}\right)+\pi\left(a_{i j}\right)\right\}=\left|\pi\left(\frac{x_{i}}{x_{j}}\right)-\pi\left(a_{i j}\right)\right|$ is quasiconvex and strictly quasiconvex, i.e., both its lower and strict lower level sets are convex.

By definition, if $x^{*}$ is efficient then $x^{*}$ is also locally efficient. Conversely, given $x^{*}$, locally efficient, suppose, by contradiction, that it is not efficient. Then there exists $y \in \mathbb{R}_{++}^{N}$ such that

$$
\left|\pi\left(\frac{x_{i}^{*}}{x_{j}^{*}}\right)-\pi\left(a_{i j}\right)\right| \geq\left|\pi\left(\frac{y_{i}}{y_{j}}\right)-\pi\left(a_{i j}\right)\right| \quad \forall i, j,
$$


with at least one inequality strict. Since the function is (strictly) quasiconvex, this property also holds for any $z$ in the open segment with endpoints $x^{*}$ and $y$, and, in particular, for $z$ arbitrarily close to $x^{*}$. This contradicts the assumption that $x^{*}$ is locally efficient. Hence $x^{*}$ must be efficient for $\left(X_{\pi}\right)$.

As a first conclusion, taking $\pi(t)=t$, we obtain that

Theorem $3 x^{*} \in \mathbb{R}_{++}^{N}$ is efficient for $(X)$ iff $x^{*}$ is locally efficient for $(X)$.

Theorem 4 Let $\pi: \mathbb{R}_{++} \longrightarrow \mathbb{R}$ be strictly increasing then, $x^{*} \in \mathbb{R}_{++}^{N}$ is efficient for $(X)$ iff $x^{*}$ is efficient for $\left(X_{\pi}\right)$.

\section{ProOF}

Let $x^{*}$ be an efficient solution of problem $(X)$; we will show that $x^{*}$ is also efficient for $\left(X_{\pi}\right)$. Suppose, by contradiction, that $x^{*}$ is not efficient for $\left(X_{\pi}\right)$, thus, by Lemma 2 , $x^{*}$ is not locally efficient for $\left(X_{\pi}\right)$.

Then there exists $y \in \mathbb{R}_{++}^{N}$, sufficiently close to $x^{*}$, such that

$$
\left|\pi\left(\frac{x_{i}^{*}}{x_{j}^{*}}\right)-\pi\left(a_{i j}\right)\right| \geq\left|\pi\left(\frac{y_{i}}{y_{j}}\right)-\pi\left(a_{i j}\right)\right| \quad \forall i, j,
$$

with at least one inequality strict, and satisfying

$$
\begin{aligned}
& \frac{y_{i}}{y_{j}} \geq a_{i j} \forall i, j, \text { such that } \frac{x_{i}^{*}}{x_{j}^{*}}>a_{i j} \\
& \frac{y_{i}}{y_{j}} \leq a_{i j} \quad \forall i, j, \text { such that } \frac{x_{i}^{*}}{x_{j}^{*}}<a_{i j}
\end{aligned}
$$

Moreover, (7) implies

$$
\pi\left(\frac{y_{i}}{y_{j}}\right)=\pi\left(a_{i j}\right), \quad \forall i, j \text { such that } \pi\left(\frac{x_{i}^{*}}{x_{j}^{*}}\right)=\pi\left(a_{i j}\right) .
$$

Since $\pi$ is assumed to be strictly increasing, (8) and (9) can be rephrased as

$$
\begin{aligned}
& \pi\left(\frac{y_{i}}{y_{j}}\right) \geq \pi\left(a_{i j}\right), \quad \forall i, j \text { such that } \pi\left(\frac{x_{i}^{*}}{x_{j}^{*}}\right)>\pi\left(a_{i j}\right), \\
& \pi\left(\frac{y_{i}}{y_{j}}\right) \leq \pi\left(a_{i j}\right), \quad \forall i, j \text { such that } \pi\left(\frac{x_{i}^{*}}{x_{j}^{*}}\right)<\pi\left(a_{i j}\right) .
\end{aligned}
$$

By (7) this implies that

$$
\left|\frac{y_{i}}{y_{j}}-a_{i j}\right| \leq\left|\frac{x_{i}^{*}}{x_{j}^{*}}-a_{i j}\right| \quad \forall i, j,
$$

with at least one inequality strict. This contradicts the assumption that $x^{*}$ is efficient for $(X)$. 
The converse, is shown analogously using Theorem 3 and will not be given here.

Taking $\pi(t)=\log t$, we have that $x^{*} \in \mathbb{R}_{++}^{N}$ is efficient for $(X)$ iff $x^{*}$ is efficient for $\left(X_{\log }\right)$,

$$
\min _{x \in \mathbb{R}_{++}^{N}}\left(\left|\log \left(x_{i}\right)-\log \left(x_{j}\right)-\log \left(a_{i j}\right)\right|\right)_{i \neq j}
$$

For a given $x \in \mathbb{R}_{++}^{N}$ let $\log (x)$ denote the vector

$$
\log (x)=\left(\log \left(x_{1}\right), \log \left(x_{2}\right), \ldots, \log \left(x_{N}\right)\right) .
$$

The discussion above shows the following

Corollary $5 x^{*} \in \mathbb{R}_{++}^{N}$ is efficient for $(X)$ iff $\log \left(x^{*}\right)$ is efficient for the piecewise linear convex vector-optimization problem $(Y)$,

$$
\min _{y \in \mathbb{R}^{N}}\left(\left|y_{i}-y_{j}-\log \left(a_{i j}\right)\right|\right)_{i \neq j} .
$$

Corollary 6 The set of efficient solutions of $(X)$ is connected.

ProOF

By $\underline{3}$, the set $E_{Y}$ of efficient solutions of $(Y)$ is connected. Using Corollary 5 one has that the set of efficient solutions of $(X)$ is the image of $E_{Y}$ under the continuous mapping

$$
\left(z_{1}, \ldots, z_{N}\right) \mapsto\left(\exp \left(z_{1}\right), \ldots, \exp \left(z_{N}\right)\right)
$$

showing connectedness.

Corollary 5 shows that, in particular, any optimal solution to

$$
\min _{y \in \mathbb{R}^{N}} \sum_{i, j=1}^{N}\left|y_{i}-y_{j}-\log \left(a_{i j}\right)\right|^{2}
$$

is efficient for $(Y)$, yielding

Corollary 7 The row geometric mean $x^{*}$,

$$
x_{i}^{*}=\left(\prod_{j=1}^{N} a_{i j}\right)^{\frac{1}{N}}, i=1, \ldots, N
$$

is efficient for $(X)$

Proof

Optimality conditions, which are both necessary and sufficient for the unconstrained convex smooth program (13) read

$$
\sum_{j \neq k}\left(y_{k}-y_{i}-\log \left(a_{k j}\right)\right)-\sum_{i \neq k}\left(y_{i}-y_{k}-\log \left(a_{i k}\right)\right)=0, \quad k=1, \ldots, N .
$$


Since $A$ is a positive reciprocal matrix, $\log \left(a_{i k}\right)=-\log \left(a_{k i}\right)$ for all $i, k$, thus 15 . can also be written as

$$
N y_{k}=\sum_{j=1}^{N} y_{j}+\sum_{j=1}^{N} \log \left(a_{k j}\right), \quad k=1, \ldots, N,
$$

a particular solution of which is given by $y$,

$$
y_{k}=\frac{1}{N} \sum_{j=1}^{N} \log \left(a_{k j}\right)=\log \left(\prod_{j=1}^{N} a_{k j}\right)^{\frac{1}{N}}, \quad k=1, \ldots, N
$$

Hence, $x^{*}$ defined in (14) is such that $\log \left(x^{*}\right)$ is efficient for $(Y)$. Hence, $x^{*}$ is efficient for $(X)$.

Now we present a geometrical characterization of efficiency.

Definition 8 Given $y \in \mathbb{R}^{N}$, let $G(y)$ be the digraph $G(y):=(\{1,2, \ldots, N\}, E(y))$,

$$
(i, j) \in E(y) \text { iff } i \neq j \text { and } y_{i}-y_{j} \geq \log \left(a_{i j}\right)
$$

Observe that, by definition, for $i, j$ given, $i \neq j$, either $(i, j) \in E(y)$ or $(j, i) \in$ $E(y)$, or both.

Theorem $9 y$ is efficient for $(Y)$ iff $G(y)$ is strongly connected.

\section{PROOF}

Let $y^{*}$ be an efficient solution of $(Y)$. This is equivalent, $\underline{\underline{3}}$, to the fact that $y^{*}$ is an optimal solution of the scalar problem $\left(P_{\lambda}\right)$

$$
\min _{y \in \mathbb{R}^{N}} \rho_{\lambda}(y):=\sum_{i \neq j} \lambda_{i j}\left|y_{i}-y_{j}-\log \left(a_{i j}\right)\right|
$$

for some $\lambda=\left(\lambda_{i j}\right)_{i \neq j}$, with $\lambda_{i j}>0$ for all $i, j, i \neq j$. Problem $\left(P_{\lambda}\right)$ is convex, hence, a necessary and sufficient optimality condition for $y^{*}$ is

$$
\mathbf{0} \in \partial \rho_{\lambda}\left(y^{*}\right) .
$$

The objective of $\left(P_{\lambda}\right)$ can be written as

$$
\rho_{\lambda}(y)=\sum_{i \neq j} \lambda_{i j} \max \left\{\left(y_{i}-y_{j}-\log \left(a_{i j}\right)\right),\left(y_{j}-y_{i}+\log \left(a_{i j}\right)\right)\right\} .
$$

hence, every subgradient $\xi$ at $y^{*}$ has the form

$$
\begin{aligned}
\xi= & \sum_{\substack{(i, j) \in E\left(y^{*}\right) \\
(j, i) \notin E\left(y^{*}\right)}} \lambda_{i j}\left(\mathbf{e}^{i}-\mathbf{e}^{j}\right)+\sum_{\substack{(i, j) \notin E\left(y^{*}\right) \\
(j, i) \in E\left(y^{*}\right)}} \lambda_{i j}\left(-\mathbf{e}^{i}+\mathbf{e}^{j}\right)+ \\
& +\sum_{\substack{(i, j) \in E\left(y^{*}\right) \\
(j, i) \in E\left(y^{*}\right)}} \lambda_{i j}\left[\mu_{i j}\left(\mathbf{e}^{i}-\mathbf{e}^{j}\right)+\left(1-\mu_{i j}\right)\left(-\mathbf{e}^{i}+\mathbf{e}^{j}\right)\right]
\end{aligned}
$$


where $\mu_{i j} \in\left[0, \underline{1]}\right.$ for all $i, j$ such that $\{(i, j),(j, i)\} \subset E\left(y^{*}\right)$. Hence, condition 16 can be rewritten as

$$
0=\sum_{\substack{(i, j) \in E\left(y^{*}\right) \\(j, i) \notin E\left(y^{*}\right)}} \lambda_{i j}+\sum_{\substack{(i, j) \notin E\left(y^{*}\right) \\(j, i) \in E\left(y^{*}\right)}}-\lambda_{i j}+\sum_{\substack{i, j) \in E\left(y^{*}\right) \\(j, i) \in E\left(y^{*}\right)}} \lambda_{i j}\left[\mu_{i j}-\left(1-\mu_{i j}\right)\right] \forall i,
$$

that is

$$
0=\sum_{(i, j) \in E\left(y^{*}\right)} \hat{\lambda}_{i j}-\sum_{(j, i) \in E\left(y^{*}\right)} \hat{\lambda}_{j i} \forall i=1,2, \ldots, N
$$

where $\hat{\lambda}_{i j}>0$, for all $i, j$ with $i \neq j$.

The homogeneous system (19) has at least one positive solution if and only if there exists a feasible flow in $G\left(y^{*}\right)$ verifying the lower bound on the flow $\hat{\lambda}_{i j} \geq 1$, for every $\operatorname{arc}(i, j)$. Following the circulation theorem of Hoffman [8] 11, this is equivalent to the non existence of cuts $(S, \bar{S})$ having positive value $V(S)$, where

$$
V(S)=d(S)+l(S, \bar{S})-u(\bar{S}, S),
$$

and $d(S)$ is the sum of the demands at nodes of $S, l()$ and $u()$ are the sums of lower and upper bounds on the corresponding arcs. In our problem every demand is null, then $d(S)=0$. Moreover

$$
l(S, \bar{S})=\#\left\{(i, j) \in G\left(y^{*}\right): i \in S, j \notin S\right\},
$$

that is, $l(S, \bar{S})$ is the number of arcs from $S$ to its complement. On other hand, there is no upper bounds on the individual flows through the arcs, that is $u(\bar{S}, S)=+\infty$ if $(\bar{S}, S) \neq \emptyset$.

Hence, $G\left(y^{*}\right)$ cannot contain directed cuts, i.e. cuts satisfying $(\bar{S}, S)=\emptyset$, 18,11 , since in other case, there exists $S$ such that $u(\bar{S}, S)=0$ which implies a positive value of $V(S)$ in 200 . Finally, note that a directed graph is strongly connected if and only if it has no directed cuts.

From the previous results one then obtains

Corollary 10 Vector $x \in \mathbb{R}_{++}^{N}$ is efficient for $(X)$ iff $G(\log (x))$ is strongly connected.

Corollary 11 If $x \in \mathbb{R}_{++}^{N}$ is efficient for $(X)$ and $x^{*} \in \mathbb{R}_{++}^{N}$ is such that $E\left(\log \left(x^{*}\right)\right) \supseteq$ $E(\log (x))$, then $x^{*}$ is efficient for $(X)$.

Remark 12 A characterization similar to that obtained in Corollary 10 is possible for weakly efficient solutions. We recall that $x^{*} \in \mathbb{R}_{++}^{N}$ is said to be weakly efficient for $(X)$ iff no $x \in \mathbb{R}_{++}^{N}$ exists with

$$
\left|\frac{x_{i}}{x_{j}}-a_{i j}\right|<\left|\frac{x_{i}^{*}}{x_{j}^{*}}-a_{i j}\right| \quad \text { for all } i, j, i \neq j
$$

With the same scheme of the proof, one can show that $x^{*} \in \mathbb{R}_{++}^{N}$ is weakly efficient for $(X)$ iff $G\left(\log \left(x^{*}\right)\right)$ contains at least one cycle. 
Corollary 10 will be the cornerstone of a geometrical characterization of the efficient set for $(X)$. First we have

Lemma 13 Given $y^{*} \in \mathbb{R}^{N}$, the following statements are equivalent:

1. $y^{*}$ is efficient for $(Y)$.

2. For all $k=1, \ldots, N$, the set $B_{k}\left(y^{*}\right):=\left\{z \in \mathbb{R}^{N}: z_{k}=0, z_{i}-z_{j}-\log \left(a_{i j}\right) \geq 0\right.$ $\forall i, j=1, \ldots, N$ such that $\left.y_{i}^{*}-y_{j}^{*}-\log \left(a_{i j}\right) \geq 0\right\}$ is bounded.

ProOF

$y^{*}$ is efficient for $(Y)$ iff for all $k=1, \ldots, N,\left(y_{1}^{*}, \ldots, y_{k-1}^{*}, y_{k+1}^{*}, \ldots, y_{N}^{*}\right)$ is efficient for $\left(Y_{k}\right)$

$$
\min \left(\left|y_{i}-y_{j}-\log \left(a_{i j}\right)\right|_{i \neq j, j \neq k},\left|y_{j}+\log \left(a_{k j}\right)\right|_{j \neq k},\left|y_{i}-\log \left(a_{i k}\right)\right|_{i \neq k}\right)
$$

$\left(Y_{k}\right)$ is a linear multiobjective regression problem with design matrix of maximum rank, $N-1$ (it contains an $(N-1) \times(N-1)$ identity submatrix). Hence, Theorem 1 of [3] applies. Thus, $y^{*}$ is efficient iff the sets $B_{k}\left(y^{*}\right)$ are bounded

Theorem 14 For $x^{*} \in \mathbb{R}_{++}^{N}$ define $C\left(x^{*}\right)$ as

$$
C\left(x^{*}\right):=\left\{x \in \mathbb{R}_{+}^{N}: x_{i}-a_{i j} x_{j} \geq 0, \forall i, j=1, \ldots, N \text { such that } x_{i}^{*}-a_{i j} x_{j}^{*} \geq 0\right\}
$$

The following statements are equivalent:

1. $x^{*}$ is efficient for $(X)$.

2. For all $k=1, \ldots, N$, the set $\left\{x \in C\left(x^{*}\right): x_{k}=1\right\}$ is contained in $\mathbb{R}_{++}^{N} \cup\{0\}$.

3. $C\left(x^{*}\right) \subseteq \mathbb{R}_{++}^{N} \cup\{0\}$.

\section{ProOF}

$(1 \Rightarrow 2)$ Let $x^{*}$ be an efficient solution for $(X)$ and $k \in\{1, \ldots, N\}$. Then the vector $x^{k}=\frac{1}{x_{k}^{*}} x^{*}$ is also efficient for $(X)$, thus by Corollary $5 \log x^{k}$ is efficient for Problem $(Y)$. By Lemma 13 the set

$$
\begin{aligned}
B_{k}\left(\log x^{k}\right)= & \left\{z \in \mathbb{R}^{N}: z_{k}=0, z_{i}-z_{j}-\log \left(a_{i j}\right) \geq 0\right. \\
& \left.\forall i, j=1 \ldots N \text { such that } \log \left(x_{i}^{*}\right)-\log \left(x_{j}^{*}\right)-\log \left(a_{i j}\right) \geq 0\right\} \\
= & \left\{z \in \mathbb{R}^{N}: z_{k}=0, z_{i}-z_{j}-\log \left(a_{i j}\right) \geq 0\right. \\
& \left.\forall i, j=1 \ldots N \text { such that } x_{i}^{*}-x_{j}^{*} a_{i j} \geq 0\right\}
\end{aligned}
$$

is bounded.

Let us suppose by contradiction that there exists $x \in C\left(x^{*}\right) \cap\left\{x: x_{k}=1\right\}$, with at least one null component, say $x_{1}=0$. 
Since $x^{*} \in \mathbb{R}_{++}^{N}$, the vector $x^{\lambda}:=(1-\lambda) x+\lambda x^{*} \in \mathbb{R}_{++}^{N}, \forall \lambda \in(0,1]$ and $x^{\lambda} \in$ $C\left(x^{*}\right) \cap\left\{x: x_{k}=1\right\}$ by convexity of such set. Then $\log x^{\lambda} \in B_{k}\left(\log x^{*}\right), \forall \lambda \in(0,1]$. Since $B_{k}\left(\log x^{*}\right)$ is bounded, the limit

$$
\lim _{\lambda \downarrow 0} \log \left(x_{1}^{\lambda}\right)
$$

is finite, which is a contradiction with the assumption that $x_{1}=0$.

( $2 \Rightarrow 3$ ) Let $x \in C\left(x^{*}\right)$ and suppose, by contradiction, that at least one of its components, say $x_{i}$, is zero. Since $x \neq 0$, there exists at least a nonzero component $x_{k}$. Since $C\left(x^{*}\right)$ is a polyhedral cone, the vector $\frac{1}{x_{k}} x \in C\left(x^{*}\right)$ and has a zero component which contradicts the assumption that $C\left(x^{*}\right) \cap\left\{x: x_{i}=1\right\} \subseteq \mathbb{R}_{++}^{N} \cup\{0\}$.

$(3 \Rightarrow 1)$ Let us assume that $C\left(x^{*}\right) \subseteq \mathbb{R}_{++}^{N} \cup\{0\}$, and we will show that $B_{k}\left(\log x^{*}\right)$ is bounded.

$C\left(x^{*}\right)$ can be re-written as

$$
C\left(x^{*}\right)=\left\{\sum_{d \in D} \lambda_{d} x^{d}: \lambda_{d} \geq 0 \forall d \in D\right\}
$$

where $\left\{x^{d}: d \in D\right\}$ is the (finite) set of extreme directions of $C\left(x^{*}\right)$.

Since, by assumption, $C\left(x^{*}\right) \subseteq \mathbb{R}_{++}^{N} \cup\{0\}$, one has that $x^{d}$ has all its components strictly positive (else, since $0 \in C\left(x^{*}\right)$, one would have some nonzero $x \in C\left(x^{*}\right) \backslash$ $\left.\mathbb{R}_{++}^{N}\right)$. Hence, for each $k=1, \ldots, N$,

$$
C\left(x^{*}\right) \cap\left\{x \in \mathbb{R}^{N}: x_{k}=1\right\}=\left\{\sum_{d \in D} \frac{\lambda_{d}}{x_{k}^{d}} x^{d}: \lambda_{d} \geq 0, \sum_{d \in D} \lambda_{d}=1\right\} .
$$

Thus $C\left(x^{*}\right) \cap\left\{x \in \mathbb{R}^{N}: x_{k}=1\right\}$ is bounded (it is the convex combination of a finite set of points). Hence, there exist $0<L_{1}^{k} \leq U_{1}^{k}, \ldots, 0<L_{N}^{k} \leq U_{N}^{k}$ such that $L_{i}^{k} \leq x_{i} \leq U_{i}^{k}, \forall i=1, \ldots, N, \forall x \in C\left(x^{*}\right) \cap\left\{x \in \mathbb{R}^{N}: x_{k}=1\right\}$. Given $z \in B_{k}\left(\log x^{*}\right)$, the vector $e^{z}:=\left(\exp \left(z_{1}\right), \ldots, \exp \left(z_{k-1}\right), 1, \exp \left(z_{k+1}\right), \ldots, \exp \left(z_{N}\right)\right)$, satisfies

$$
\frac{\exp \left(z_{i}\right)}{\exp \left(z_{j}\right)}=\exp \left(z_{i}-z_{j}\right) \geq \exp \left(\log \left(a_{i j}\right)\right)=a_{i j} \forall i, j \text { such that }(i, j) \in E\left(\log x^{*}\right),
$$

thus

$$
e^{z} \in C\left(x^{*}\right) \cap\left\{x \in \mathbb{R}^{N}: x_{k}=1\right\} .
$$

Hence, we have $0<L_{i}^{k} \leq \exp \left(z_{i}\right) \leq U_{i}^{k}$, thus $-\infty<\log \left(L_{i}^{k}\right) \leq z_{i} \leq \log \left(U_{i}^{k}\right)<$ $+\infty \forall i$, showing that $B_{k}\left(\log x^{*}\right)$ is bounded. By Lemma $13 \log \left(x^{*}\right)$ is efficient for $(Y)$ thus $x^{*}$ is efficient for $(X)$.

We summarize with the following

Corollary 15 Let $\mathcal{E}=\{E \subseteq\{1, \ldots, N\} \times\{1, \ldots, N\}$ such that $\forall i, j, i \neq j,(i, j) \in$ $E$ or $(j, i) \in E\}$ and, for each $E \in \mathcal{E}$, define $C_{E}$ as

$$
C_{E}=\left\{x \in \mathbb{R}_{+}^{N}: x_{i}-a_{i j} x_{j} \geq 0 \forall(i, j) \in E\right\} .
$$

Then, for $x^{*} \in \mathbb{R}_{++}^{N}$, the following statements are equivalent: 
1. $x^{*}$ is efficient for $(X)$.

2. There exists $E \in \mathcal{E}$ such that $C_{E} \subseteq \mathbb{R}_{++}^{N} \cup\{0\}$ satisfying $x^{*} \in C_{E}$.

3. There exists $E \in \mathcal{E}$ such that $(\{1, \ldots, N\}, E)$ is strongly connected satisfying $x \in C_{E}$.

Proof

$(1 \Rightarrow 2,3)$. Set $E=E\left(\log \left(x^{*}\right)\right)$, thus $C_{E}=C\left(x^{*}\right)$ and $G\left(\log \left(x^{*}\right)\right)=(\{1, \ldots, N\}, E)$. Since $x^{*} \in C\left(x^{*}\right)$, Part 2 follows from Theorem 14 and Part 3 from Corollary 10.

$(2 \Rightarrow 1)$ Let $E \in \mathcal{E}$ such that $C_{E} \subseteq \mathbb{R}_{++}^{N} \cup\{0\}$ satisfies $x^{*} \in C_{E}$. By definition $C\left(x^{*}\right) \subseteq C_{E}$, thus, by Theorem 14 one has that $x^{*}$ is efficient.

$(3 \Rightarrow 1)$ Let $E \in \mathcal{E}$ such that $(\{1, \ldots, N\}, E)$ is strongly connected and $x^{*} \in C_{E}$. By definition, $E\left(\log \left(x^{*}\right)\right) \supseteq E$, thus, $G\left(\log \left(x^{*}\right)\right)$ is strongly connected. Finally, Corollary 10 implies that $x^{*}$ is efficient.

\section{The Eigenvector Method and efficiency}

In this Section we show, by means of an example, that the solution provided by the Eigenvector Method may not be efficient for $(X)$. To do this, consider the $4 \times 4$ matrix $A$,

$$
A=\left(\begin{array}{cccc}
1 & 2 & 6 & 2 \\
\frac{1}{2} & 1 & 4 & 3 \\
\frac{1}{6} & \frac{1}{4} & 1 & \frac{1}{2} \\
\frac{1}{2} & \frac{1}{3} & 2 & 1
\end{array}\right)
$$

The eigenvalues of $A$ are the roots of a polynomial function of fourth degree. Hence, they can be calculated analytically. In particular, using the symbolic computation package MAPLE $\underline{\underline{12}}$ highest-modulus eigenvalue $\lambda_{\max }$,

$$
\lambda_{\max } \approx 4.103141140
$$

From $\lambda_{\max }$, an associated eigenvector $x$ is obtained exactly. In order to obtain the corresponding $x$-graph, observe that for $i \neq j$,

$$
(i, j) \in E((\log (x))) \text { iff } \Delta(x)_{i j}:=x_{i}-x_{j} a_{i j} \geq 0
$$

The coefficients $\Delta(x)_{i j}$ were calculated numerically using interval arithmetic, accommodating round-off errors, using the package INTPAK [6]. The results are displayed in Table 1

This yields

$$
E(\log (x))=\{(1,3),(1,4),(2,1),(2,3),(4,2),(4,3)\}
$$

No directed path from 3 to 1 . Hence, $x$ is not efficient. 


\begin{tabular}{|c|c|c|}
\hline$i$ & $j$ & $\Delta(x)_{i j}$ \\
\hline 1 & 2 & $(-2.5066080075891861670,-2.5066080075891843904)$ \\
1 & 3 & $(.14380576758662222 \mathrm{e}-1, .14380576758667095 \mathrm{e}-1)$ \\
1 & 4 & $(1.871897356839027781,1.871897356839037799)$ \\
2 & 1 & $(1.2533040037945921952,1.2533040037945930835)$ \\
2 & 3 & $(.2604942921739238855, .2604942921739260512)$ \\
2 & 4 & $(-1.953230537705527812,-1.953230537705517857)$ \\
3 & 1 & $(-.23967627931111824 \mathrm{e}-2,-.23967627931103703 \mathrm{e}-2)$ \\
3 & 2 & $(-.651235730434815128 \mathrm{e}-1,-.651235730434809715 \mathrm{e}-1)$ \\
3 & 4 & $(-.356208049799086164 \mathrm{e}-1,-.356208049799073180 \mathrm{e}-1)$ \\
4 & 1 & $(-.935948678419518899,-.935948678419513890)$ \\
4 & 2 & $(.651076845901839286, .651076845901842604)$ \\
4 & 3 & $(.712416099598146361 \mathrm{e}-1, .712416099598172328 \mathrm{e}-1)$ \\
\hline
\end{tabular}

Table 1: Coefficients $\Delta(x)_{i j}$

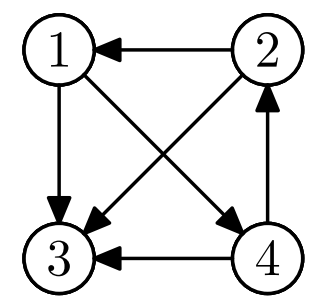

Figure 1: The graph $E(\log (x))$

\section{References}

[1] J. Barzilai. Deriving weights from pairwise comparison matrices. Journal of the Operational Research Society, 48:1226-1232, (1997).

[2] N. Bryson. A goal programming method for generating priority vectors. Journal of the Operational Research Society, 46:641-648, (1995).

[3] E. Carrizosa, E. Conde, M. Fernández, F.R. Muñoz, and Puerto. J. Paretooptimality in linear regression. Journal of Mathematical Analysis and Applications, 190:129-141, (1995).

[4] V. Chankong and Y. Haimes. Multiobjective Decision Making. North-Holland, Amsterdam, 1983.

[5] Expert Choice. at http://www.expertchoice.com.

[6] A. Connell and R.M. Corless. An experimental interval arithmetic package in Maple. Interval Computations, 2:120-134, (1993). 
[7] W.D. Cook and M. Kress. Deriving weights from pairwise comparison ratio matrices: An axiomatic approach. European Journal of Operational Research, 37(3):355-362, (1988).

[8] A.J. Hoffman. Some recent applications of the theory of linear inequalities to extremal combinatorial analysis. in: Proceedings of Symposia in Applied Mathematics R.Bellman and M. Hall, J.r., (Eds.), American Mathematical Society, Providence, RI, 10:113-127, 1960.

[9] F.A. Lootsma. A model for the relative importance of the criteria in the multiplicative ahp and smart. European Journal Of Operational Research, 94:467476, (1996).

[10] B. Martos. Nonlinear Programming. Theory and methods. North-Holland, Amsterdam, 1975.

[11] S.T. McCormick. How to compute least infeasible flows. Mathematical Programming, Series B, 78(2):179-194, (1997).

[12] M. Monagan, K. Geddes, K. Heal, G. Labahn, and S. Vorkoetter. Maple V Programming Guide for Release 5. Springer-Verlag, New York, 1997.

[13] R. Ramanathan. A note on the use of goal programming for the multiplicative ahp. Journal of Multi-criteria Decision Analysis, 6:296-307, (1997).

[14] T.L. Saaty. A scaling method for priorities in hierarchical structures. Journal of Mathematical Psychology, 15:234-281, (1977).

[15] T.L. Saaty. Multicriteria Decision Making: The Analytic Hierarchy Process. McGraw-Hill, New York, 1980.

[16] T.L. Saaty. Eigenvector and logaritmic least squares. European Journal of Operational Research, 48:156-160, (1990).

[17] T.L. Saaty. Fundamentals of Decision Making. RSW Publications, Pittsburg, PA, 1994.

[18] T.L. Saaty. How to make a decision: The analytic hierarchy process. Interfaces, 24:19-43, (1994).

[19] S. Schaible. Fractional programming. in: Handbook of Global Optimization R. Horst and P.M. Pardalos, Eds., pages 495-608, 1995.

[20] R. Steuer. Multiple criteria optimization: Theory, Computation, Application. Wiley, New York, 1986.

[21] M. Zeleny. Multiple Criteria Decision Making. McGraw-Hill, New York, 1982. 